Wege für einen ausgewogenen Ausbau erneuerbarer Energien

\title{
100 Prozent sind machbar, Herr Nachbar!
}

\section{Der Ausbau der Erneuerbaren Energien hat gerade erst begonnen. Auch wenn zu- künftige Anlagen sich in Deuschland harten Naturschutzkriterien stellen müssen, ist das Potenzial zur regenerativen Stromerzeugung noch weitgehend uner- schöpft. Die Deckung des gesamten Strombedarfs aus Erneverbaren ist möglich.}

$\mathrm{U}$ Von Wolfram Krewitt, Joachim Nitsch und Guido Reinhardt m die drängenden Nachhaltigkeitsdefizite unserer Energieversorgung zu mildern, hat sich die Bundesregierung das Ziel gesetzt, den Beitrag erneuerbarer Energien bis 2010 zu verdoppeln. Ausgehend von diesem Einstieg in den Ausbau erneuerbarer Energien erfordern die langfristig angestrebten Klimaschutzziele wie die Kohlendioxid-Reduktion um 50 Prozent bis 2050 allerdings, dass erneuerbare Energien bis zur Mitte des Jahrhunderts zur Hauptenergiequelle der Energieversorgung werden und bis dahin etwa die Hälfte des Energiebedarfs decken.

Trotz der enormen Unterstützung, die erneuerbare Energien in der öffentlichkeit erfahren, zeigt beispielsweise die Entwicklung der Windkraft in den letzten Jahren, dass dieser Sympathie Grenzen gesetzt sind, wenn die Aspekte des Natur- und Landschaftsschutzes bei den Bemühungen zur Kohlendioxid-Einsparung möglicherweise nicht ausreichend gewürdigt werden.

Der lange und durchaus beschwerliche Weg von heute drei Prozent Anteil bis hin zur Deckung des halben Energiebedarfs durch erneuerbare Energie wird nur gangbar sein, wenn Klima-, Landschafts- und Naturschutz sich nicht als Gegensätze gegenüberstehen, sondern sich als ergänzende Orientierungspunkte für einen verantwortungsvollen Ausbau erneuerbarer Energien etablieren. Im Auftrag des Bundesumweltministeriums (BMU) wurden dazu im Forschungsprojekt ,Ökologisch optimierter Ausbau der Nutzung erneuerbarer Energien in Deutschland" Wege aufgezeigt, auf denen der Ausbau erneuerbarer Energien sowohl den Anforderungen des Klimaschutzes als auch des Natur- und Landschaftsschutzes gleichermaßen gerecht wird (1).

\section{- Erneverbare Energien und Naturschutz}

So sehr Kohlendioxid-Emissionen als handlicher Indikator für die Klimaverträglichkeit die Experten zum Optimieren zukünftiger Energieversorgungssystem (ver-)leiten, so schwierig ist es, die Vielschichtigkeit der von kleinräumigen Eigenarten geprägten Landschafts- und Naturschutzbelange im Rahmen von Langfristszenarien adäquat zu erfassen.

Um die spezifischen Aspekte des Naturschutzes, die auf der Ebene einzelner Anlagen im Rahmen der Umweltverträglichkeitsprüfung untersucht werden, auch bei der Entwicklung von Szenarien für ein gesamtes Energiesystem perspektivisch zu berïcksichtigen, wurde in der BMU-Studie in einem „NaturschutzPlus“-Szenario ein Potenzial abgeleitet, das langfristig unter strengen naturschutzfachlichen Anforderungen für die Nutzung erneuerbarer Energien zur Verfügung steht. Eine eher vorsichtige Festlegung von Potenzialen reflektiert die Erkenntnis, dass neue Technologien - auch wenn sie wie die erneuerbaren Energien generell eine hohe Wertschätzung genießen sich in behutsamer und sensibler Weise ausbreiten sollten. Die generell bereits hohe Belastung unserer Lebensräume durch technische Bauwerke ist vielfach mit einem subjektiven Unbehagen gegen weitere Eingriffe verbunden, das sich in erster Linie gegen die „Neuankömmlinge" wendet, auch wenn diese mit geringeren ökologischen Auswirkungen als bereits vorhandene Anlagen verbunden sind. Zur Beibehaltung der hohen Akzeptanz der erneuerbaren Energien sollte daher der Grundsatz gelten, gegebenenfalls auf lokale Potenziale zu verzichten, wenn zu große Konflikte mit deren Ausnutzung verbunden sein sollten.

\section{- Energiequellen}

Der Themenkomplex Wasserkraft und Naturschutz wurde in einer Expertenanhörung mit Vertretern der relevanten Interessengruppen erörtert. Übereinstimmend konnte festgestellt werden, dass weder die „kleine“ oder ,große“ Wasserkraft per se zu einer ökologisch verträglicheren Form der Wasserkraftnutzung führt. Tendenziell ist allerdings davon auszugehen, dass für kleine Anlagen die Aufwendungen zur Einhaltung ökologischer Mindestanforderungen mit anteilmäßig höheren Kosten als bei großen Anlagen verbunden sind. Dies wirkt sich auf die Wirtschaftlichkeit kleiner Anlagen aus, die häufig schon durch höhere Stromerzeugungskosten gekennzeichnet sind.

In der Expertenrunde bestand ein weitgehender Konsens darüber, dass die in Deutschland noch verbleibenden ungenutzten beziehungsweise weitgehend naturnahen Gewässer, deren Anteil auf circa zehn Prozent der Fließgewässer geschätzt wird, für einen Neubau von Wasserkraftanlagen nicht in Frage kommen sollten. Das sowieso nur noch kleine Ausbaupotenzial der Wasserkraftnutzung wird dadurch nur unwesentlich eingeschränkt. Das größte Ausbaupotenzial kann durch die Modernisierung und Erweiterung bestehender großer und kleiner Anlagen unter ökologischer Aufwertung erschlossen werden.

Allein wegen ihrer Größe und der Notwendigkeit, windgünstige Randbedingungen zu nutzen, führen Windenergieanlagen zu einer Veränderung des Landschaftsbildes. Je nach Ausstattung der Landschaft können sie dabei landschaftliche Merkmale „hoher Eigenart und Schönheit" stören, wodurch ein nach dem Bundesnaturschutzgesetz zu schützendes Gut beeinträchtigt würde. Untersuchungen über die Auswirkungen von Windenergieanlagen auf die Avifauna zeigen, dass verschiedene Vogelarten unterschiedliche Reaktionen bezüglich ihres Zug-, Rast- und Brutverhaltens zeigen: während „Kulturfolger“ kaum Reaktionen zeigen, wird das Verhalten von Arten, die eine hohe Empfindlichkeit gegenüber anthropogenen Lebensraumveränderungen haben, merklich beeinflusst. Anhand detaillierter Analysen in den Beispielregionen BadenWürttemberg und Niedersachsen konnte gezeigt werden, dass das Potenzial der Windenergienutzung durch Restriktionen des Natur- und Landschaftsschutzes zwar wie erwartet stark eingeschränkt wird, dass aber andererseits trotz strenger naturschutzfachlicher Anforderungen noch ein beträchtliches Potenzial zum Ausbau der Windenergie zur Verfügung steht. Die Ergebnisse deuten darauf 
hin, dass nicht primär die direkten Eingriffe der Windenergienutzung in Naturschutzbelange der limitierende Faktor sind, sondern letztendlich die gesellschaftliche Akzeptanz den weiteren Ausbau der Windenergie begrenzen wird.

Um die erhöhte gesellschaftliche Sensibilität gegenüber einem weiteren Ausbau der Windenergienutzung an Land perspektivisch zu berücksichtigen, wurde im Szenario NaturschutzPlus unterstellt, dass zukünftig rund ein halbes Prozent der Fläche in Deutschland für die Windenergienutzung zur Verfügung stehen wird, wobei die Anteile in den Küstenregionen größer und im Binnenland deutlich kleiner sind. Das Potenzial liegt damit bei insgesamt etwa 20.000 bis 25.000 Megawatt, wobei ein großer Teil des verbleibenden Ausbaupotenzials vor allem durch das ,Repowering“ an bestehenden Standorten erschlossen werden kann.

Bei einem Ausbau der Windenergienutzung auf See ist eine besondere Berücksichtigung der Belange des Natur-, Umwelt- und Meeresschutzes geboten. Wegen der fehlenden Erfahrungen sind bisher die Kenntnisse über mögliche Einflüsse von OffshoreWindparks auf die Meeresökologie noch unvollständig. Nach einem Abgleich verschiedener Nutzungsinteressen sind von der Bundesregierung erste konfliktarme Flächen identifiziert worden, die unter den gegenwärtigen Bedingungen für die Startphase und die erste Ausbauphase als besondere Eignungsgebiete für Offshore-Windparks in Betracht kommen. Da es sich bei der Windenergienutzung auf See um einen großflächigen und langfristigen Eingriff in die Meeresumwelt handelt, soll zur Wahrung des Vorsorgeprinzips ein stufenweiser Ausbau realisiert werden.

Bioenergieträger können aus einer Vielzahl biogener Ressourcen bereitgestellt werden, die vor allem der Land- und Forstwirtschaft entstammen, aber auch aus anderen Bereichen wie aus der Landschaftspflege, industriellen Prozessen oder Haushalten. Grundsätzlich schließen sich Naturschutz und Biomassebereitstellung nur auf sehr wenigen Kernflächen des Naturschutzes vollständig aus. Die Nutzung von Reststoffen wird durch Naturschutzaspekte kaum berührt.

Die Möglichkeit zum Anbau von Energiepflanzen wird durch die verfügbaren Flächenpotenziale begrenzt. In der BMU-Studie wurde untersucht, welchen Einfluss Nachhaltigkeitsziele der Bundesregierung wie ,Ausweitung des Ökolandbaus“, „Bereitstellung von Flächen zum Biotopverbund gemäß Paragraphen 3 und 5 des Bundesnaturschutzgesetzes“ und „Bepflanzen aller erosionsgefährdeten Ackerstandorte mit mehrjährigen Kulturen“ gegenüber einer Politik ausüben, die diese Ziele nicht oder nur teilweise verfolgt.

Bei der Umsetzung der genannten Ziele stehen 2010 rund 2,3 Millionen Hektar und 2050 rund 1,9 Millionen Hektar Ackerlandflächen weniger für den Anbau von Biomasse zur Verfügung, als es unter weniger restriktiven Bedingungen der Fall wäre. Im Jahr 2010 kämen demnach nur relativ marginale Flächen um 0,2 Millionen Hektar für einen Energiepflanzenanbau in Frage. Diese steigen über die folgenden Dekaden jedoch insbesondere auf Grund der Bevölkerungsentwicklung und der Ertragssteigerung in der Landwirtschaft deutlich an und liefern langfristig auch unter strengen Naturschutzkriterien mit 4,2 Millionen Hektar ein nennenswertes Biomassepotenzial.

Andererseits ergeben sich aus diesen Restriktionen zusätzliche Potenziale auf Naturschutzflächen, da auch auf vielen dem Naturschutz zugeführten Flächen Biomasse produziert werden kann. Durch die Biomasseabfuhr von aus Naturschutzsicht wünschenswerten Flächen ergeben sich inklusive der Biomasse aus dem Anbau mehrjähriger Pflanzen auf erosionsgefährdeten Standorten immerhin circa $150 \mathrm{PJ} / \mathrm{a}$ zusätzliche Mengen an Biomasse, dieses Potenzial entspricht der gesamten Biogasmenge.

Die zur Verfügung stehende Biomasse kann entweder stationär zur Erzeugung von Strom und Wärme oder zur Herstellung von Kraftstoffen verwendet werden. Das energiebezogene Potenzial des Anbaus von Biomasse ist für die stationäre Strom- und Wärmeerzeugung etwa doppelt so groß wie ihre Nutzung für die Herstellung von Biokraftstoffen. Das Potenzial der Reststoffe ändert sich im Laufe der Zeit nicht wesentlich, während das Potenzial für Anbaubiomasse bis zum Jahr 2050 deutlich steigt. Hierdurch wird auch ein möglicher Zeitpfad für den Ausbau des Biomassemarktes im Kraftstoffbereich vorgegeben. Wird von den hier dargestellten strengen Restriktionen ausgegangen, so kann das EU Ziel von 5,75 Prozent Biokraftstoffanteil im Jahr 2010 auf der Basis von Anbaubiomasse auf den zur Verfügung stehenden Flächen bei Weitem nicht erreicht werden. Erst mittel- bis langfristig stehen ausreichende Flächen für Energiepflanzen zur Verfügung.

Bau und Betrieb von gebäudeintegrierten Fotovoltaikanlagen und solarthermischer Kollektoren sind aus Naturschutzsicht unproblematisch. Kontrovers diskutiert wird dagegen der Bau von großen Freiflächenanlagen. Einerseits ist der Bau von Freiflächenanlagen keinesfalls mit einer Flächenversiege-

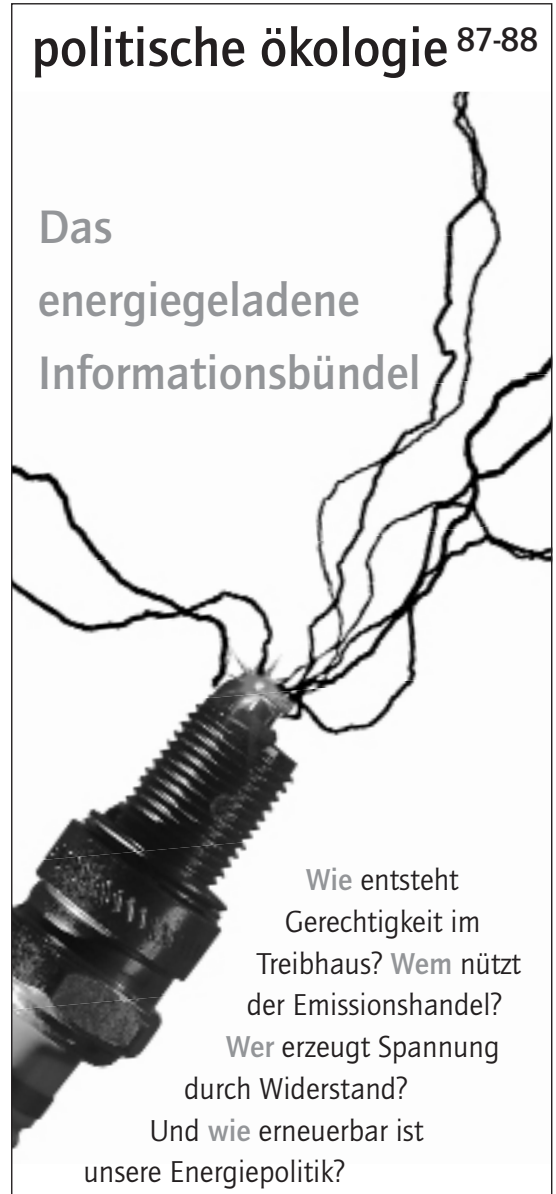

Lesen Sie, worüber vor und hinter den Kulissen der Energie- und Klimapolitik diskutiert wird.

Mit Beiträgen von H. Breyer, H. Graßl, C. Bals, J. Morgan, M. Jänicke, F. Matthes, H. E. Ott,

K. Pachauri, H. Scheer u.v.m.

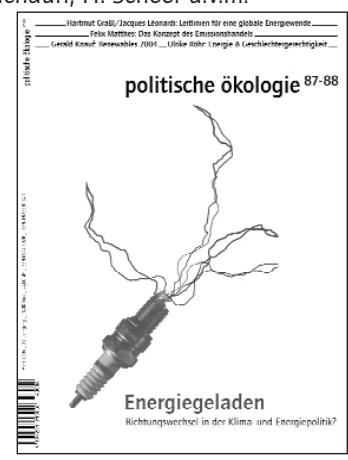

Stillen Sie Ihre Neugier!

$$
\begin{aligned}
& \text { _Fordern Sie Heft 87-88 an } \\
& \text { _-15,- } € \text { (inkl. Versand) } \\
& \text { _ISBN 3-936581-37-1 } \\
& \text { _oekom verlag } \\
& \text { _Fax 089/544 184 } 49 \\
& \text { _www.oekom.de }
\end{aligned}
$$




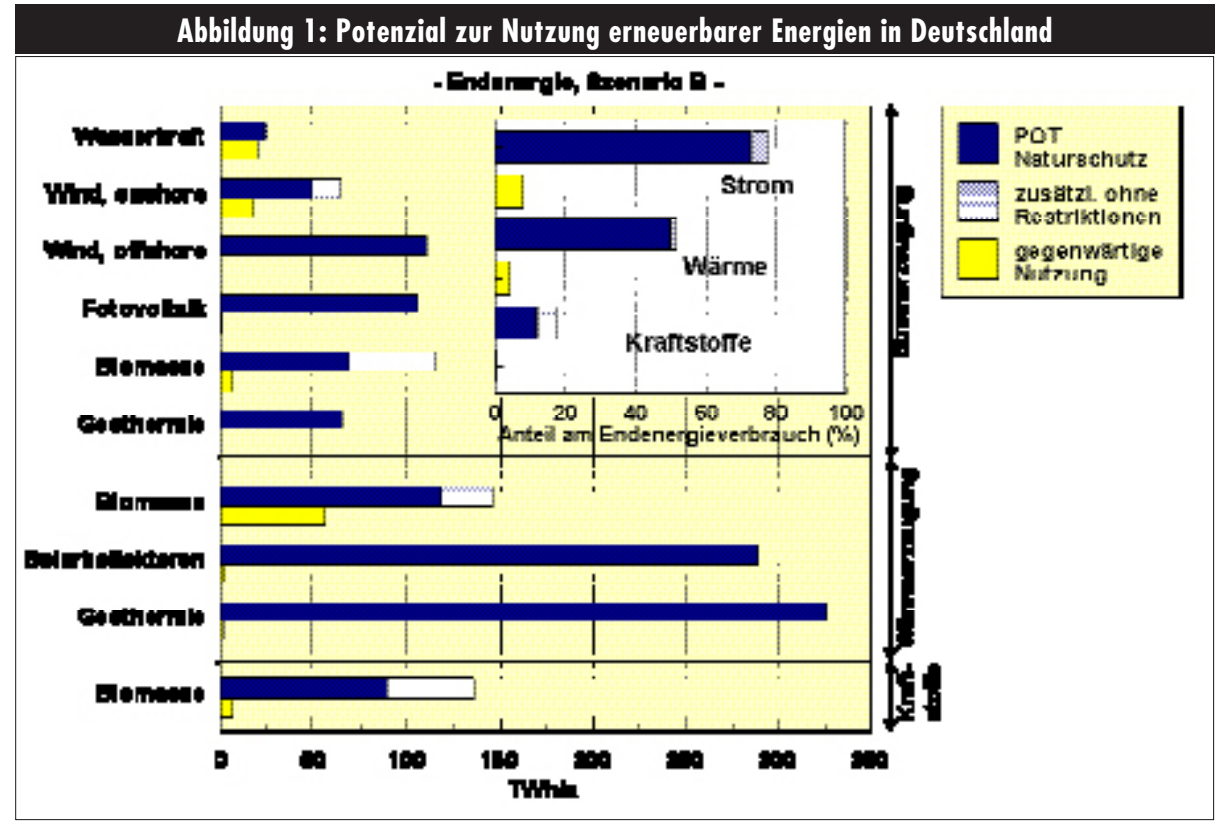

Quelle: Eigene Darstellung

lung gleichzusetzen. Untersuchungen weisen darauf hin, dass die biologische Vielfalt auf Grünland mit Solarmodulen höher ist als auf einem agrarindustriell genutzten Acker. Andererseits wird durch eine Fotovoltaikanlage auf einer Freifläche immer ein Eingriff in das Ökosystem verursacht und stellt einen zusätzlichen Flächenbedarf dar, der aus Naturschutzsicht möglichst zu vermeiden ist.

Auch in den ambitionierten Szenarien zum Ausbau erneuerbarer Energien wird das Potenzial, das innerhalb bestehender Siedlungsstrukturen zur Verfügung steht, bei Weitem nicht ausgenutzt, so dass aus Kapazitätsgründen die Belegung von Freiflächen nicht notwendig ist. Es ist abzusehen, dass durch große Freiflächenanlagen das Problem der Beeinflussung des Landschaftsbildes zunehmend eine Rolle spielen wird und sich damit auch hier - ähnlich wie bei der Windenergie - eine Akzeptanzdiskussion in der Gesellschaft einstellen kann. Durch eine Beschränkung auf gebäudeintegrierte Anlagen kann dies vermieden werden. Außerdem bleibt durch diese Beschränkung der Anreiz bestehen, weiterhin die Konzepte der Integration der Fotovoltaik in Gebäuden und anderen Siedlungsbauwerken voranzubringen. Aus industriepolitischer Sicht mag die Installation von Freiflächenanlagen eine wichtige Rolle für die schnelle und kostengünstige Ausweitung der PVMarktvolumina spielen. Allerdings sollten Freiflächen nur unter strengen Kriterien und im Sinne einer Überbrückungsstrategie während der Markteinführung der Fotovoltaik genutzt werden. Mittelfristig sollte schon aus Akzep- tanzgründen auf die Nutzung landwirtschaftlicher Flächen für die Fotovoltaik verzichtet werden.

\section{- Strenge Naturschutzkriterien}

Potenziale erneuerbarer Energien in Deutschland sind auch unter strengen Naturschutzkriterien ausreichend, um den gesamten Energiebedarf zu decken. Strenge Anforderungen aus Sicht des Naturund Landschaftsschutzes reduzieren zwar für einzelne erneuerbare Energien, insbesondere für die Nutzung der Windenergie und der Biomasse, das verfügbare Potenzial. Gegenüber einem „BasisSzenario“, das von den technisch-strukturellen Potenzialen erneuerbarer Energien unter Berücksichtigung wesentlicher Belange des Naturschutzes wie Ausschluss von Windparks in Naturschutzgebieten oder Vorgaben des Gewässerschutzes bei der Wasserkraftnutzung ausgeht, wird das insgesamt in Deutschland zur Verfügung stehende Potenzial durch zusätzliche strenge naturschutzfachliche Anforderungen allerdings nur unwesentlich eingeschränkt ( siehe Abb. 1). Weder das langfristige Kohlendioxid-Minderungziel noch das Ausbauziel müssen in Frage gestellt werden. Wird auf den aus naturschutzfachlicher Sicht kritisch einzuschätzenden kurzfristig forcierten Einsatz von Biokraftstoffen aus Anbaubiomasse verzichtet, so können auch die Kosten für den Ausbau erneuerbare Energien reduziert werden. Bei gleicher Kohlendioxid-Minderung führen langfristig die strengen naturschutzfachlichen Kriterien im Vergleich zum „Basis-Szenario“ zu etwas höheren Kosten, da durch die Einschränkungen bei der Windenergie und Biomassenutzung vergleichsweise teurere Technologien wie Fotovoltaik größere Beiträge zur Energieversorgung leisten müssen.

\section{- 100 Prozent möglich}

Da das große Potenzial erneuerbarer Energien in Deutschland bisher nur ansatzweise genutzt wird, weisen fast alle Sparten erneuerbarer Energien auch unter naturschutzfachlichen Restriktionen noch ganz beträchtliche Ausbaupotenziale auf. Bereits bei einem Bezug auf heutige Verbrauchswerte könnten beträchtliche Anteile des Energiebedarfs Deutschlands durch erneuerbare Energien gedeckt werden. Berücksichtigt man die noch hohen Potenziale einer Effizienzsteigerung in allen Verbrauchsbereichen, die im Zuge einer nachhaltigen Entwicklung mobilisiert werden können, so zeigt sich die Tragfähigkeit einer Ausbaustrategie erneuerbarer Energien noch deutlicher. Längerfristig können auch die Importpotenziale von Strom aus erneuerbaren Energien im Zuge einer europäischen Gesamtstrategie der Mobilisierung erneuerbarer Energien zur Deckung der deutschen Energienachfrage genutzt werden. Auch Wasserstoff kann aus erneuerbaren Energien bereitgestellt und insbesondere als Treibstoff im Verkehr eingesetzt werden. Einer langfristig hundertprozentigen Deckung der Energienachfrage mittels erneuerbarer Energien steht also auch bei strengen naturschutzfachlichen Restriktionen aus Potenzialsicht nichts entgegen.

\section{Anmerkung}

(1) Nitsch, J. / Krewitt, W. / Reinhardt, G. / Pehnt, M. / Fischedick, M. et al.: Ökologisch optimierter Ausbau der Nutzung erneuerbarer Energien in Deutschland. Berlin 2004.

\section{Die Autoren}

Dr. Wolfram Krewitt ist Projektleiter, Dr. Joachim Nitsch Leiter der Abteilung Systemanalyse und Technikbewertung am Deutschen Zentrum für Luft- und Raumfahrt (DLR). Dr. Guido Reinhardt ist wissenschaftlicher Mitarbeiter beim, Institut für Energie- und Umweltforschung (ifeu). Kontakt: DLR, Pfaffenwaldring 38-40, 70569 Stuttgart Tel. 0711-6862766, E-Mail: wolfram.krewitt@dlr.de, ifeu, Wilckensstr. 3, 69120 Heidelberg. E-Mail: guido.reinhardt@ifeu.de 
(c) 20I0 Authors; licensee IÖW and oekom verlag. This is an article distributed under the terms of the Creative Commons Attribution Non-Commercial No Derivates License (http://creativecommons.org/licenses/by-nc-nd/3.o/), which permits unrestricted use, distribution, and reproduction in any medium, provided the original work is properly cited. 